\title{
O USO DE PROGRAMAÇÃO MATEMÁTICA PARA PROVA DA TEORIA DAS VANTAGENS COMPARATIVAS E A BALANÇA COMERCIAL
}

\author{
Sdepan Bogosian Neto \\ Centro de Projetos de Navios - Marinha do Brasil \\ Ilha das Cobras, Ed. 16, s/no. Centro, Rio de Janeiro CEP 20091-000 \\ e-mail: sbogosian@gmail.com
}

\begin{abstract}
RESUMO
Este artigo trata de uma demonstração alternativa da Teoria das Vantagens Comparativas, desta vez com o uso de programação matemática (método simplex) a $n$ países e $p$ produtos. $\mathrm{Na}$ demonstração o trabalho é o único insumo. Com os resultados do mesmo modelo, e com o uso de um modelo matemático simplificado para o comércio internacional, é demonstrado que quanto mais tecnologia empregada, mais favorável a balança comercial.

Palavras-chave: Balança Comercial, Fronteiras de Possibilidade de Produção, Teoria das Vantagens Comparativas, Ricardo.
\end{abstract}

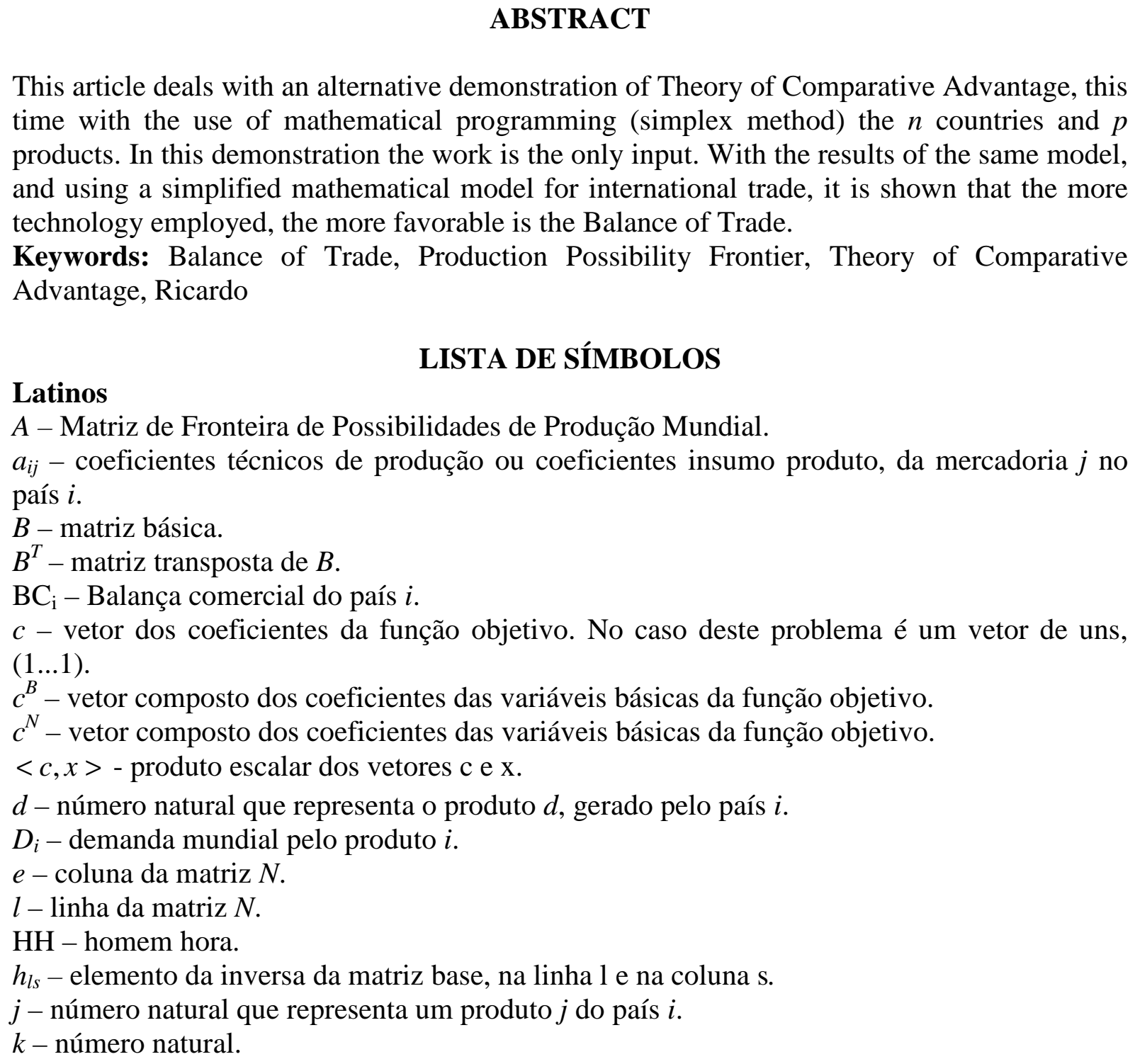

This article deals with an alternative demonstration of Theory of Comparative Advantage, this time with the use of mathematical programming (simplex method) the $n$ countries and $p$ products. In this demonstration the work is the only input. With the results of the same model, and using a simplified mathematical model for international trade, it is shown that the more technology employed, the more favorable is the Balance of Trade.

Keywords: Balance of Trade, Production Possibility Frontier, Theory of Comparative Advantage, Ricardo

\section{Latinos}

\section{LISTA DE SÍMBOLOS}

$A$ - Matriz de Fronteira de Possibilidades de Produção Mundial.

$a_{i j}$ - coeficientes técnicos de produção ou coeficientes insumo produto, da mercadoria $j$ no país $i$.

$B$ - matriz básica.

$B^{T}$ - matriz transposta de $B$.

$\mathrm{BC}_{\mathrm{i}}-$ Balança comercial do país $i$.

$c$ - vetor dos coeficientes da função objetivo. No caso deste problema é um vetor de uns, (1...1).

$c^{B}$ - vetor composto dos coeficientes das variáveis básicas da função objetivo.

$c^{N}$ - vetor composto dos coeficientes das variáveis básicas da função objetivo.

$<c, x\rangle$ - produto escalar dos vetores $\mathrm{c} \mathrm{e} \mathrm{x}$.

$d$ - número natural que representa o produto $d$, gerado pelo país $i$.

$D_{i}$ - demanda mundial pelo produto $i$.

$e$ - coluna da matriz $N$.

$l$ - linha da matriz $N$.

$\mathrm{HH}$ - homem hora.

$h_{l s}$ - elemento da inversa da matriz base, na linha 1 e na coluna s.

$j$ - número natural que representa um produto $j$ do país $i$.

$k$ - número natural. 
$l$ - linha da matriz $N$.

$L_{i}$ - reserva de trabalho do país i.

$l_{i j}$ - quantidade de trabalho do país i utilizada no produto $\mathrm{j}$.

$n$ - número natural que representa a quantidade de países.

$N$ - matriz não-básica.

$N^{T}$ - matriz transposta de $N$.

$p$ - numero natural representante do número de produtos.

$P_{j}$ - população de um país $j$.

$R$ - relação entre o número de pessoas (consumidores ou trabalhadores, conforme o caso) e os homens hora de um país. Considerado constante para todos os países. Dimensão HH/no. Pessoas.

$t_{i j}$ - tecnologia empregada na mercadoria $j$ no país $i$, considerada um número adimensional.

$x$ - vetor dos produtos produzidos por cada país do mundo.

$x_{i j}$ - quantidade produzida da mercadoria $j$ no país $i$.

\section{Gregos}

$\kappa_{i j}$ - consumo do insumo $j$ no país $i$.

$\gamma_{j}-$ consumo per capita do produto $\mathrm{j}$.

$\lambda_{i j}$ - constante que relaciona o país i e o produto j a uma quantidade de trabalho empregada, ou seja, número de pessoas empregadas no país i para produzir $\mathrm{j}$.

\section{LISTA DE ABREVIATURAS E SIGLAS}

FPP - fronteira de possibilidade de produção.

PPL - programa de programação linear.

\section{INTRODUÇÃO}

A globalização é um fenômeno contextualizado em diferentes planos (BOGOSIAN NETO, 2010, p. 7): econômico; político; cultural; social; ideológico (BARBOSA, 2008, p.8, os três primeiros planos).

No plano econômico, tema deste artigo, Souza (2009, p. 134) descreve a globalização como "[...] a abolição das fronteiras econômicas entre as nações que permitiria a livre mobilidade de capitais, mercadorias, tecnologias e força de trabalho em escala mundial".

Uma vez livres para comerciar, as forças econômicas fariam com que algumas regiões, com afinidades para produção satisfatória de alguns produtos, passassem a produzi-los de modo eficiente e se integrassem ao comércio internacional (OHMAE, 1996), com ganho para todos.

A ideia da produção eficiente e consequente integração ao comércio internacional não é nova em economia, remontando aos clássicos, como Ricardo (1817) e nela reside a importância do tema do artigo: a Teoria das Vantagens Comparativas.

Ricardo (1817) explica (e mostra numericamente) que, para o comércio internacional valer a pena para um país, basta haver vantagens comparativas (Teoria das Vantagens Comparativas) e não vantagens absolutas, ou seja, basta o país conseguir fazer mais facilmente um bem que outro, e não necessariamente fazer seu produto melhor que qualquer outro país. Neste caso, o país deve especializar-se na produção do produto que já é bom, e realizar trocas. Estas trocas fazem com que, não somente este país, mas o mundo saia com um ganho (Williamson, 1988).

A natureza do conceito de vantagens comparativas consiste num problema de otimização, na medida em que o comércio internacional, como explicado por Ricardo "aumenta" ou "otimiza" a produção com a adoção da especialização, ou seja, trata-se de uma busca de maximizar algo, no caso a produção. Neste sentido, Dorfman, Samuelson e Solow (1987, p. 31 e seguintes), por exemplo, usam a programação linear para provar o proposto por Ricardo, contudo, sem se utilizar da teoria do valor-trabalho. 
A especialização, entretanto, tem trazido aos países que exportam commodities (SINGER-PREBISCH ..., 1999 e SARKAR, 1986) uma capacidade sempre decrescente de importação de bens de maior valor agregado (Embora, em alguns períodos, haja uma inversão desta tendência como no "boom de commodities de 2000", (ibid.)).

O desenvolvimento que se segue tenta estender a prova de Ricardo e explorar seus limites (dentro da Teoria do Valor-Trabalho) e também mostrar porque os países exportadores commodities teriam um menor desempenho em sua balança comercial.

\section{DESENVOLVIMENTO}

Uma fronteira de possibilidade de produção (FPP) designa o lugar geométrico das quantidades máximas de cada bem que um país pode produzir segundo sua dotação de fatores, conforme Carvalho e Silva (2007, p. 33).

$$
\begin{aligned}
& L_{1}=a_{11} x_{11}+a_{12} x_{12}+\ldots+a_{1 p} x_{1 p} \\
& L_{2}=a_{21} x_{21}+a_{22} x_{22}+\ldots+a_{2 p} x_{2 p} \\
& \ldots \\
& L_{n}=a_{n 1} x_{n 1}+a_{n 2} x_{n 2}+\ldots+a_{n p} x_{n p}
\end{aligned}
$$

Como o único insumo é o trabalho, chama-se de $L_{i}$ o montante de todo o trabalho do país $i$. Considerando-se $n$ países, pode-se organizar um sistema linear em que cada linha representa a FPP de um país na forma representada no sistema (1). Este sistema é uma extrapolação da reta apresentada por Williamson (1988, p.23), generalizada em um hiperplano.

$$
\begin{gathered}
\text { O sistema representado por (1) pode ser escrito na forma matricial (2). } \\
\left(\begin{array}{l}
L_{1} \\
L_{2} \\
\ldots \\
L_{n}
\end{array}\right)=\left(\begin{array}{cccccccccccc}
a_{11} & a_{12} & \ldots & a_{n 1} & 0 & 0 & \ldots & 0 & 0 & 0 & \ldots & 0 \\
0 & 0 & \ldots & 0 & a_{21} & a_{22} & \ldots & a_{2 n} & 0 & 0 & \ldots & 0 \\
0 & 0 & \ldots & 0 & 0 & 0 & \ldots & 0 & 0 & 0 & \ldots & 0 \\
0 & 0 & \ldots & 0 & 0 & 0 & \ldots & 0 & a_{n 1} & a_{n 2} & \ldots & a_{n n}
\end{array}\right)\left(\begin{array}{c}
x_{11} \\
x_{12} \\
\ldots \\
x_{1 n} \\
x_{21} \\
x_{22} \\
\ldots \\
x_{2 n} \\
\ldots \\
x_{n 1} \\
x_{n 2} \\
\ldots \\
x_{n n}
\end{array}\right)
\end{gathered}
$$

Criando a função de produção mundial, ou seja, toda produção de todas as mercadorias por todos os países do mundo, representada por (3): 


$$
z=\left(\begin{array}{lll}
1 & \ldots & 1
\end{array}\right)\left(\begin{array}{c}
x_{11} \\
x_{12} \\
\ldots \\
x_{1 n} \\
x_{21} \\
x_{22} \\
\ldots \\
x_{2 n} \\
\ldots \\
x_{n 1} \\
x_{n 2} \\
\ldots \\
x_{n n}
\end{array}\right)=<c, x>
$$

Pode-se formular o programa de programação linear (PPL) da forma:

$\max z=\langle c, x\rangle$

sujeito a

$$
\begin{aligned}
& \left(\begin{array}{cccccccccccc}
a_{11} & a_{12} & \ldots & a_{n 1} & 0 & 0 & \ldots & 0 & 0 & 0 & \ldots & 0 \\
0 & 0 & \ldots & 0 & a_{21} & a_{22} & \ldots & a_{2 n} & 0 & 0 & \ldots & 0 \\
0 & 0 & \ldots & 0 & 0 & 0 & \ldots & 0 & 0 & 0 & \ldots & 0 \\
0 & 0 & \ldots & 0 & 0 & 0 & \ldots & 0 & a_{n 1} & a_{n 2} & \ldots & a_{n n}
\end{array}\right)\left(\begin{array}{c}
x_{11} \\
x_{12} \\
\ldots \\
x_{1 n} \\
x_{21} \\
x_{22} \\
\ldots \\
x_{2 n} \\
\ldots \\
x_{n 1} \\
x_{n 2} \\
\ldots \\
x_{n n}
\end{array}\right)=\left(\begin{array}{c}
L_{1} \\
L_{2} \\
\ldots \\
L_{n}
\end{array}\right)
\end{aligned}
$$

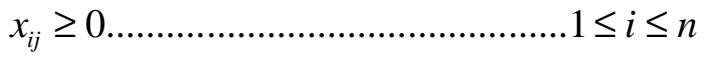

$$
\begin{aligned}
& 1 \leq j \leq n
\end{aligned}
$$

Ou na forma compacta, dita canônica:

$$
\begin{aligned}
& \max z=\langle c, x> \\
& A x=b
\end{aligned}
$$

A equação (5) é em programação linear conhecida como função objetivo. Nela há o produto escalar do vetor $c$ pelo vetor $x$.

A equação (6) representa as restrições do PPL, neste caso são todas as FPP dos países.

O sistema composto pelas equações (5) e (6) também pode ser reescrito na forma das equações (7) e (8).

$$
\begin{aligned}
& \max z=<c, x> \\
& B x^{B}+N x^{N}=b
\end{aligned}
$$

A nova forma de representação separa a matriz $A \in R^{n x m}$ nas submatrizes $B \in R^{n x n}$ e $N \in R^{n x m}:$ a matriz base multiplicada pelo vetor de variáveis básicas, $x^{B} \in R^{n}$; e a 
matriz não-básica multiplicada pelo vetor de variáveis não básicas, $x^{N} \in R^{m}$. Isto está expresso na equação (9).

$$
A=[B \vdots N]
$$

O vetor $x$ é por sua vez composto dos vetores $x^{B}$ e $x^{N}$, conforme (10).

$$
x=\left[\begin{array}{l}
x^{B} \\
\cdots \\
x^{N}
\end{array}\right]
$$

A definição de matriz base (YOSHIDA, 1987, p. 23) é a submatriz B com determinante diferente de zero.

A "solução básica", $\bar{x}$, por sua vez, é definida como a solução em que todas as variáveis não básicas sejam zero, conforme (11).

$$
\bar{x} \equiv\left[\begin{array}{l}
x^{B} \\
\ldots \\
x^{N}
\end{array}\right]=\left[\begin{array}{l}
x^{B} \\
\ldots \\
0
\end{array}\right]=\left[\begin{array}{l}
B^{-1} b \\
\ldots \\
0
\end{array}\right]
$$

A "solução básica", $\bar{x}$, por sua vez, é definida como a "solução básica viável" se além de satisfazer (11), ainda $\bar{x} \geq 0$.

Prova-se que uma solução ótima ${ }^{1}$ é uma "solução básica viável” de um PPL (para maiores detalhes ver capítulo 3 de Yoshida (1987), p. 41 e 42).

\section{MÉTODO² SIMPLEX}

Segundo Yoshida (1987, p. 44) o método simplex determina as soluções ótimas de um PPL, caso existam, ou permite concluir que o problema é ilimitado ou inviável. ${ }^{3}$ saber:

O algoritmo simplex (YOSHIDA, 1987, p. 45 e p. 46), é constituído de 5 passos a

a) Passo 1: Início. Determinação de solução básica viável;

b) Passo 2: Critério de Optimalidade;

c) Passo 3: Critério de Entrada;

d) Passo 4: Critério de Saída;

e) Passo 5: Retorno. Voltar para o Passo 2.

\section{CRITÉRIO DE OPTIMALIDADE}

O critério de optimalidade é o passo em que se verifica se o vetor de variáveis básicas é ótimo, ou seja, é a solução básica viável do PPL, que maximiza a função objetivo.

Para melhor explicar o critério de optimalidade, apresentam-se alguns desenvolvimentos. Assim, rearranjando a equação (8), pode-se obter:

$$
x^{B}=B^{-1} b-B^{-1} N x^{N}
$$

Com base em (12), a função objetivo pode ser reescrita na forma (13):

$$
\begin{aligned}
& <c, x>=<c^{B}, B b^{-1}>+<\gamma, x^{N}> \\
& \gamma=c^{N}-N^{T}\left(B^{-1}\right)^{T} c^{B}
\end{aligned}
$$

O critério de optimalidade consiste em verificar se $\gamma$ é menor que zero, caso em que a solução é ótima (YOSHIDA, 1987, p.45). Isto pode facilmente ser entendido com a simples observação da equação (13), em que $\gamma$ pertence à função objetivo. Se fizermos na solução do problema qualquer $x^{N} \geq 0$, então a função objetivo diminuirá de valor, pois o

\footnotetext{
${ }^{1}$ Procurando uma explicação mais intuitiva, solução ótima é a solução melhor a que se pode chegar. Pensando em produção, seria o máximo de produtos que se poderiam obter com o uso dos insumos descritos nas restrições, matriz A.

${ }^{2}$ Em Yoshida (1987, p. 44) a palavra algoritmo serve para descrever os passos do método simplex.

${ }^{3}$ Ilimitado - soluções maximizantes podem crescer indefinidamente (YOSHIDA, 1987, p. 11). Inviável - o conjunto de pontos que satisfazem às restrições é vazio (YOSHIDA, 1987, p. 67).
} 
produto $<\gamma, x^{N}><0$. Assim a solução $x^{B}=B^{-1} b$ só pode ser ótima no caso em que $\gamma \leq 0$. (YOSHIDA, 1987, p. 46).

\section{CRITÉRIOS DE ENTRADA, SAÍDA E RETORNO}

Estes critérios auxiliam na condução do problema numérico, mas não apresentam interesse para este trabalho, portanto não serão aqui apresentados. Para maiores detalhes sobre eles, deve-se consultar Yoshida (1987, p. 45 e 46).

\section{PROVA DE QUE A ESPECIALIZAÇÃO CONSTITUI A SOLUÇÃO ÓTIMA DO PPL REPRESENTA E ESTA A MAIOR QUANTIDADE DE MERCADORIA PASSÍVEL DE SER PRODUZIDA PELO MUNDO}

Com o uso dos passos expostos em Yoshida (1987, p. 45 e 46), busca-se provar que a solução ótima do PPL representa que a especialização da produção conduz ao máximo de produção de cada mercadoria e, portanto, que a soma de todas as mercadorias produzidas por todos os países, é maximizada, o que representa a solução ótima do problema de PPL.

Observando o sistema (4), a matriz A pode ser reescrita nas suas submatrizes $B$ e $N$. Sem perda de generalidade, estabelece-se que o país 1 tenha vantagem comparativa no produto 1, e o país 2 tenha no produto 2 , assim sucessivamente o produto $\mathrm{n}$ no país $\mathrm{n}$, de forma que o vetor de variáveis básicas seja constituído pelas variáveis $x_{i i}$.

Dizer que um país tenha vantagem comparativa na produção de um produto significa dizer que:

$$
\frac{a_{i d}}{a_{i i}} \geq 1, \forall d \neq i, d=1, \ldots, n, i=1, \ldots, n, j=1, \ldots, n
$$

Assim de acordo com esta escolha, seria dizer que:

$$
\frac{a_{i d}}{a_{i i}} \geq 1, \forall d \neq i, d=1, \ldots, n, i=1, \ldots, n
$$

Assim rearranjando o sistema (4), tem-se:

$$
\begin{aligned}
& \max z=<c^{B}, x^{B}>+<c^{N}, x^{N}> \\
& \text { sujeito a } \\
& \left(\begin{array}{cccccccccccc}
a_{11} & 0 & \ldots & 0 & 0 & a_{12} & \ldots & 0 & 0 & 0 & \ldots & a_{1 n} \\
0 & a_{22} & \ldots & 0 & a_{21} & 0 & \ldots & a_{2 n} & 0 & 0 & \ldots & 0 \\
0 & 0 & \ldots & 0 & 0 & 0 & \ldots & 0 & 0 & 0 & \ldots & 0 \\
0 & 0 & \ldots & a_{n n} & 0 & 0 & \ldots & 0 & a_{n 1} & a_{n 2} & \ldots & 0
\end{array}\right)\left(\begin{array}{c}
x_{11} \\
x_{22} \\
\ldots \\
x_{n n} \\
x_{21} \\
x_{12} \\
\ldots \\
x_{2 n} \\
\ldots \\
x_{n 1} \\
x_{n 2} \\
\ldots \\
x_{1 n}
\end{array}\right)=\left(\begin{array}{c}
L_{1} \\
L_{2} \\
\ldots \\
L_{n}
\end{array}\right)
\end{aligned}
$$

Desse modo as matrizes $B$ e $N$ ficam assim definidas:

$$
B=\left(\begin{array}{cccc}
a_{11} & 0 & \ldots & 0 \\
0 & a_{22} & \ldots & 0 \\
0 & 0 & \ldots & 0 \\
0 & 0 & \ldots & a_{n n}
\end{array}\right)
$$




$$
N=\left(\begin{array}{cccccccc}
0 & a_{12} & \ldots & 0 & 0 & 0 & \ldots & a_{1 n} \\
a_{21} & 0 & \ldots & a_{2 n} & 0 & 0 & \ldots & 0 \\
0 & 0 & \ldots & 0 & 0 & 0 & \ldots & 0 \\
0 & 0 & \ldots & 0 & a_{1 n} & a_{2 n} & \ldots & 0
\end{array}\right)
$$

Observando-se a matriz B, trata-se de uma matriz diagonal. Já a disposição dos elementos da matriz $\mathrm{N}$ tem uma forma regular, dada por:

$$
\begin{aligned}
& N_{l e}=0, \quad e \neq(k-1)(n+1)+2 \\
& N_{l e}=a_{l q}, \quad n(l-1)<e \leq n l \quad e \neq(n+1)(l-1)+2 \\
& \quad q=e(n l-1) \\
& \text { caso } \quad \text { contrário } \\
& N_{l e}=0
\end{aligned}
$$

Calculando a inversa da matriz $B$ :

$$
B^{-1}=\left(\begin{array}{cccc}
1 / a_{11} & 0 & \ldots & 0 \\
0 & 1 / a_{22} & \ldots & 0 \\
0 & 0 & \ldots & 0 \\
0 & 0 & \ldots . & 1 / a_{n n}
\end{array}\right)
$$

A observação de (22) permite concluir que a transporta da inversa é igual a inversa, ou seja:

$$
\left(B^{-1}\right)^{\mathrm{T}}=B^{-1}
$$

Assim a matriz será substituída por outra da forma

$$
\left(B^{-1}\right)^{T}=\left(\begin{array}{cccc}
h_{11} & 0 & \ldots & 0 \\
0 & h_{22} & \ldots & 0 \\
0 & 0 & \ldots & 0 \\
0 & 0 & \ldots & h_{n n}
\end{array}\right) \in R^{n x n}
$$

Construindo a matriz $N^{T}$ :

$$
N^{T}=\left(\begin{array}{cccc}
0 & a_{21} & \ldots & 0 \\
a_{12} & 0 & \ldots & 0 \\
0 & \ldots & \ldots & 0 \\
0 & a_{2 n} & \ldots & 0 \\
\ldots & & & \\
0 & 0 & \ldots & a_{n 1} \\
0 & 0 & \ldots & a_{n 2} \\
\ldots & \ldots & \ldots & \ldots \\
a_{1 n} & 0 & \ldots & 0
\end{array}\right) \in R^{n(n-1) x n}
$$

Calculando o produto $N^{T}\left(B^{-1}\right)^{T}$ :

$$
N^{T}\left(B^{-1}\right)^{T}=\left(\begin{array}{cccc}
0 & \frac{a_{21}}{a_{22}} & \ldots & 0 \\
\frac{a_{12}}{a_{11}} & 0 & \ldots & 0 \\
0 & \ldots & \ldots & 0 \\
0 & \frac{a_{2 n}}{a_{22}} & \ldots & 0 \\
\ldots & & & \\
0 & 0 & \ldots & \frac{a_{n 1}}{a_{n n}} \\
0 & 0 & \ldots & \frac{a_{n 2}}{a_{n 2}} \\
\ldots & \ldots & \ldots & \ldots \\
\frac{a_{1 n}}{a_{11}} & 0 & \ldots & 0
\end{array}\right) \in R^{n(n-1) x n}
$$


Assim os elementos do produto $N^{T}\left(B^{-1}\right)^{T}$, podem ser expressos pela regra geral:

$$
N^{T}\left(B^{-1}\right)^{T}=N_{e l} h_{l s}=\left\{\begin{array}{lc}
\frac{a_{1 l}}{a_{11}}, & e=(n+1)(l-1)+2 \\
\frac{a_{q l}}{a_{l l}}, & q=e-n(l-1) \quad n(l-1)<e \leq n l \\
0, \text { caso } & \text { contrário }
\end{array}\right.
$$

$1 \leq l \leq n$

$1 \leq e \leq n(n-1)$

Calculando o produto $N^{T}\left(B^{-1}\right)^{T} c^{B}$ :

$$
N^{T}\left(B^{-1}\right)^{T} c^{B}=\left(\begin{array}{cccc}
0 & \frac{a_{21}}{a_{22}} & \ldots & 0 \\
\frac{a_{12}}{a_{11}} & 0 & \ldots & 0 \\
0 & \ldots & \ldots & 0 \\
0 & \frac{a_{2 n}}{a_{22}} & \ldots & 0 \\
\ldots & & & \\
0 & 0 & \ldots & \frac{a_{n 1}}{a_{n n}} \\
0 & 0 & \ldots & \frac{a_{n 2}}{a_{n n}} \\
\ldots & \ldots & \ldots & \ldots \\
\frac{a_{1 n}}{a_{11}} & 0 & \ldots & 0
\end{array}\right)\left(\begin{array}{l}
1 \\
1
\end{array}\right)=\left(\begin{array}{c}
\frac{a_{21}}{a_{22}} \\
\frac{a_{12}}{a_{11}} \\
\ldots \\
\frac{a_{2 n}}{a_{22}} \\
\ldots \\
\frac{a_{n 1}}{a_{n n}} \\
\frac{a_{n 2}}{a_{n n}} \\
\ldots \\
\frac{a_{1 n}}{a_{11}}
\end{array}\right) \in R^{n(n-1)}
$$

Partindo, finalmente, para o cálculo de $\gamma$, tem-se e com o uso de (16):

$$
\begin{gathered}
\gamma=c^{N}-N^{T}\left(B^{-1}\right)^{T} c^{B} \\
\gamma=\left(\begin{array}{l}
1 \\
\frac{a_{21}}{a_{22}} \\
\frac{a_{12}}{a_{11}} \\
\ldots \\
\ldots
\end{array}\right)-\left(\begin{array}{c}
1-\frac{a_{21}}{a_{22}} \\
\frac{a_{2 n}}{a_{22}} \\
\ldots \\
\frac{a_{12}}{a_{11}} \\
\ldots \\
a_{n n} \\
\frac{a_{n 2}}{a_{n n}} \\
\ldots \\
\frac{a_{1 n}}{a_{11}}
\end{array}\right)=\left(\begin{array}{c}
\frac{a_{2 n}}{a_{22}} \\
\ldots \\
1-\frac{a_{n 1}}{a_{n n}} \\
1-\frac{a_{n 2}}{a_{n n}} \\
\ldots \\
1-\frac{a_{1 n}}{a_{11}}
\end{array}\right) \leq 0
\end{gathered}
$$

Procurou-se desse modo provar, matematicamente, com base num modelo relativamente mais geral que o de Williamson (1988, p.23), que realmente a especialização conduz a uma produção ótima, desde que se desconsiderem as demandas por consumo dos países.

\section{DIGRESSÃO SOBRE A DIMENSÃO DA MATRIZ A}

Em observando a Matriz A, que representa a FPP de todos os países do Mundo, percebe-se a possibilidade de ocorrência de dois casos:

(1) $\mathrm{p}=\mathrm{n}$ - cada país se especializa em um produto. A produção mundial é máxima; 
(2) $\mathrm{n}>\mathrm{p}-\mathrm{o}$ número de países é maior que o número de produtos. Assim, caso haja especialização, alguns países que não se especializarão ${ }^{4}$ em nada, pois não levam vantagem comparativa nenhuma. Estes países ficarão à margem do comércio mundial. Serão obrigados a constituir-se em autarquias;

(3) $\quad \mathrm{p}>\mathrm{n}-\mathrm{o}$ número de produtos é maior que o número de países - de modo que alguns produtos deixariam de ser produzidos, e, portanto, novamente, a demanda não seria atendida.

8 PROVA DE QUE SE A DEMANDA CONSTITUIR TAMBÉM UMA RESTRIÇÃO, A SOLUÇÃOO ÓTIMA NÃO É NECESSARIAMENTE A ESPECIALIZAÇÃO COMPLETA.

Esta demonstração será realizada para apenas 2 países e dois produtos. Introduzindo a equação de demanda, o sistema (4) altera-se para o sistema (30) $\max z=\langle c, x>$

sujeito a

$$
\left(\begin{array}{cccc}
a_{11} & a_{12} & 0 & 0 \\
0 & 0 & a_{21} & a_{22} \\
1 & 0 & 1 & 0 \\
0 & 1 & \ldots & 1
\end{array}\right)\left(\begin{array}{l}
x_{11} \\
x_{12} \\
x_{21} \\
x_{22}
\end{array}\right)=\left(\begin{array}{c}
L_{1} \\
L_{2} \\
D_{1} \\
D_{2}
\end{array}\right)
$$

Este sistema é estruturalmente diferente do anterior. Na verdade, considerando-se que as variáveis não básicas seriam os termos cruzados, neste caso, $x_{12}$ e $x_{21}$, ter-se-ia um novo sistema da forma (31):

$$
\left(\begin{array}{cccc}
a_{11} & a_{12} & 0 & 0 \\
0 & 0 & a_{21} & a_{22} \\
1 & 0 & 1 & 0 \\
0 & 1 & \ldots & 1
\end{array}\right)\left(\begin{array}{c}
x_{11} \\
0 \\
0 \\
x_{22}
\end{array}\right)=\left(\begin{array}{c}
L_{1} \\
L_{2} \\
D_{1} \\
D_{2}
\end{array}\right)
$$

Cuja solução obtida só é possível desde que $L_{1} / a_{11}$ e $L_{2} / a_{22}$ sejam respectivamente iguais a $D_{1}$ e $D_{2}$. Em qualquer outra hipótese a especialização não seria adequada de modo a resolver este PPL. Isto significa que, nestes casos, os países deveriam produzir, além dos seus bens com vantagens comparativas, outros de modo a suprir sua demanda.

Note-se que Ricardo, ao formular a teoria das vantagens comparativas, não exigia a completa especialização, mas apenas a sugeria como forma de explicar as vantagens do comércio internacional.

O modelo simplificado permite ver que a especialização pode causar também a pobreza (Bogosian Neto, 2010), pois caso, sem perda de generalidade, $D_{1} \gg L_{1} / a_{11}$, então, a produção alcançaria seu máximo, entretanto, o país 2 seria prejudicado no atendimento de sua população, pois o país 1 produz muito menos que o necessário para "alimentar" 2.

\section{PROVA DE QUE A ESPECIALIZAÇÃO TRAZ BENEFÍCIOS PARA AMBOS OS PAÍSES PARTICIPANTES DO COMÉRCIO INTERNACIONAL}

A prova de que a especialização traz benefícios para os países participantes do comércio internacional é amplamente encontrada na literatura. Entenda-se por benefícios o recebimento ou o consumo de mais bens na situação de praticantes do comércio internacional do que na situação de economias fechadas ao comércio internacional. Para maiores

\footnotetext{
${ }^{4}$ Ao se especializar completamente, um país $i$ zera o valor de todas as quantidades, $x_{i j}$, do produto $j$, exceto um único produto $k$, ou seja, faz $x_{i j}=0, j \neq k$, sendo $i, j$ e $k$ inteiros e, $x_{i j}$, real e maior ou igual a zero.
} 
esclarecimentos e consolidação desta demonstração, pode-se consultar Williamson (1987, p. 23 e seguintes) e Dorfman, Samuelson e Solow (1987, p. 31 e seguintes).

\section{TECNOLOGIA E COEFICIENTES TÉCNICOS}

Definindo a tecnologia utilizada pelo país i na produção do produto j por $t_{i j}$, este passa a ser o inverso do coeficiente de insumo produto, multiplicado pelo número de homenshora trabalhados. A figura 1 apresenta uma ilustração do coeficiente $a_{i j}$ entendido como uma função.

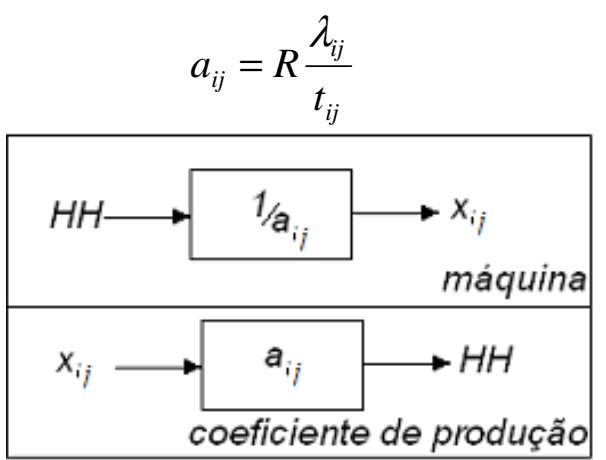

Figura 1- Dualidade máquina - coeficiente técnico de produção

A variável $\lambda_{i j}$ representa número de trabalhadores necessário para se fazer um produto i num país j. Este número deveria ser igual em qualquer país, mas razões de distribuição geográfica de fatores de produção o modificam e não inclui as mudanças tecnológicas. Neste modelo, as diferenças tecnológicas são explicitadas através da razão $\lambda_{i j} / t_{i j}$, que é diferente em cada lugar. $\mathrm{O}$ valor $R$ é o número de $\mathrm{HH}$ por trabalhador, ou seja, 8horas/dia.

Intuitivamente, entende-se que se acrescenta tecnologia para se produzir "mais" com o mesmo emprego de insumos. Esta melhoria que se obtém com a tecnologia, no caso em estudo, centra-se apenas em maior quantidade e não em maior qualidade.

Deste modo, se a tecnologia do país 1 para o produto $1, t_{11}$, é maior que aquela do país $2, t_{21}$, para o mesmo produto, $t_{11}>t_{21}$, tem-se que o trabalho para uma mesma quantidade gerada de produto 1 é menor no país $1, \frac{l_{11}}{x_{11}}<\frac{l_{21}}{x_{21}}$.

\section{CONSUMO}

A matriz de consumos é dada por $\kappa_{i j}$, que também pode ser entendida como a demanda pelo consumo do produto i, no país j. Considerando-se que:

a) os consumidores em todos os países tem as mesmas preferências, isto é, a título de exemplo, dados dois produtos, 1 e 2, e dois países 1 e 2, as proporções $\kappa_{12 /} \kappa_{11}$ e $\kappa_{22 /} \kappa_{21}$ são iguais. Portanto, há um fator de consumo per capita de cada produto é dado por $\gamma_{j}$;

b) o trabalho é proporcional à população, pelo fator $R$, idêntico para todos os países. Isto significa que o número de homens hora disponíveis depende apenas do número de trabalhadores, assim ninguém trabalha horas a mais que ninguém.

Tem-se, caso toda a população trabalhe:

$$
\kappa_{i j}=\gamma_{i} \sum_{k=1}^{p} \lambda_{k j}
$$

Definindo a população de um país $j$ por $P_{j}$, tem-se que o consumo é proporcional ao número de habitantes (no caso em que toda a população é trabalhadora, esta não é uma hipótese necessária):

$$
\kappa_{i j}=\gamma_{i} P_{j}
$$




\section{BALANÇA COMERCIAL}

A balança comercial do país i, $B C_{i}$, é dada pela diferença do que este país vende e do que compra no mercado internacional.

Desse modo, a balança comercial fica sendo, após a especialização, dada pela diferença entre o único produto do país i, $x_{i i}$, e o consumo do mesmo país, dada pela equação (35):

$$
B C_{i}=x_{i i}-\sum_{j=1}^{p} \kappa_{j i}
$$

Assim, lembrando que o sistema de equações dado por (1) ainda é válido após a especialização, a totalidade de produto do país $i$, passa a ser dada pelo estoque de trabalho sobre o coeficiente de produção, $L_{i} / a_{i i}$. O consumo do país (as compras do exterior) são, então, dados por toda a população do país $i, P_{i}$, multiplicada pelo consumo per capita,$\gamma_{j}$, como em (33). Nisto resulta (36):

$$
B C_{i}=\frac{L_{i}}{a_{i i}}-\sum_{j=1}^{p} \gamma_{j} P_{i}
$$

Substituindo na equação (35) a equação (32), tem-se:

$$
B C_{i}=\frac{L_{i} t_{i i}}{R \lambda_{i i}}-\sum_{j=1}^{p} \gamma_{j} P_{i}
$$

Como se pode ver, na equação (37), quanto maior a tecnologia maior a receita. Esta equação consegue explicar a também, grosso modo, a balança comercial positiva de países com pequena população e produtos de alto valor agregado.

\section{POSSIBILIDADE DE PERDAS COMERCIAIS PARA PAÍSES EXPORTADORES DE PRODUTOS DE ALTO VALOR AGREGADO} tecnologia.

Produtos de alto valor agregado aqui são considerados produtos intensivos em

Com o uso da equação (37) percebe-se a possibilidade de haver o empobrecimento destes países, desde que o segundo termo do membro da direita da equação (37) seja maior que o primeiro termo, o que pode ocorrer sempre que o consumo supere o ganho que traz a tecnologia empregada no estoque de trabalho para a balança comercial.

\section{CONCLUSÃO}

Procurou-se provar a Teoria das Vantagens Comparativas com o uso de programação matemática em face do entendimento de que a Teoria é de fato um problema de otimização. Neste sentido, utilizou-se o mínimo de novos conceitos, de modo a se manter fiel à formulação proposta por Ricardo. Por outro lado, isto tem o inconveniente de tornar o modelo muito restrito.

É interessante notar que, mesmo com o modelo restrito, já se alguns vislumbram problemas na especialização: se o número de países e o número de produtos forem diferentes, ou seja, se houver países a mais, há países que serão obrigados a serem autarquias; se produtos a mais, haverá escassez dos produtos que não poderão mais ser produzidos por países especializados. Além disso, percebe-se que a solução ótima pode mudar se acrescentada à restrição de que toda a demanda seja atendida.

A prova de que a balança comercial é favorável a quem tem maior economia e a quem tem maior tecnologia é também bem restrita, dependendo das hipóteses de que há uma preferência homogênea de produtos nos países e de que todos os países estão especializados. O mesmo se passa com o entendimento de quanto maior a população e, portanto o trabalho, maior será a balança comercial.

Percebe-se ser possível também a existência de um empobrecimento dos países tecnologicamente avançados sempre sua "tecnologia" não conseguir superar suas 
necessidades alimentícias (ou melhor, suas necessidades ligadas ao consumo população), embora continue válida a afirmação de que maior a tecnologia, maior a balança comercial.

\section{REFERÊNCIAS BIBLIOGRÁFICAS}

BARBOSA, A. de F. "O Mundo Globalizado: Política, Sociedade e Economia... 4a . Ed. São Paulo: Contexto, 2008.

BOGOSIAN NETO, S. "Globalização, Regionalização e Multilateralismo: desafios para os países de terceiro mundo: A globalização e as vantagens e desvantagens da Especialização da produção para os países, com base na teoria das vantagens comparativas de ricardo." Monografia apresentada à Escola de Guerra Naval como requisito parcial para recebimento do grau no curso superior. Rio de Janeiro: Escola de Guerra Naval, 2010.

CARVALHO, M. A.; SILVA, C. R. L. Economia Internacional. 4ª Ed., São Paulo: Saraiva, 2007.

DORFMAN, F. SAMUELSON, P. A., SOLOW R. M.. Linear Programming and Economic Analysis. [s.1.]: Dover, [1987?].

OHMAE, K. "O fim do Estado-Nação". Tradução de Ivo Korytowski. Rio de Janeiro: Campus, 1996.

RICARDO, D. "On the Principals of Political Enconomy and Taxation". 1817. Disponível em: http://socserv.mcmaster.ca/econ/ugcm/31l3/ricardo/Principles.pdf Acessso: 18 ago. 2010.

SARKAR, P.. The Singer-Prebisch Hypothesis: A Statistical Evaluation. Revista Cambridge Journal of Economics. [s.1.], Vol. 10, No. 4, p. 355-371, 1986. Disponível em:

<http://papers.ssrn.com/sol3/papers.cfm?abstract_id=1018568\#\#>. Acesso em: 25 ago. 2010.

SINGER-PREBISCH thesis. In: WIKIPEDIA: a enciclopédia livre. [1999?]. Disponível em: <http://en.wikipedia.org/wiki/Prebish-Singer>. Acesso em: 22 jun. 2010

SOUZA, N. A. "Economia Internacional Contemporânea: da Depressão de 1929 ao Colapso Financeiro de 2008”. São Paulo: Atlas, 2009.

WILliAMSON, J. A Economia Aberta e a Economia Mundial: Um Texto de Economia Internacional. Tradução de José Ricardo Brandão Azevedo, São Paulo: Campus, 1988.

YOSHIDA, L. K. Programação Linear. São Paulo: Atual, 1987. 